\title{
Effectiveness of group interpersonal psychotherapy for decreasing aggression and increasing social support among Chinese university students: a randomized controlled study
}

\begin{abstract}
Background: The objective of this randomized controlled experimental study was to determine the intervention effect of group interpersonal psychotherapy (G-IPT) for aggression and social support among Chinese first-grade university students. Methods: Through stratified random sampling, 1469 students, aged 18-19 years, were enrolled. Participants whose score achieved the aggressive evaluation standard were selected and then 60 participants were randomly divided into 2 groups: G-IPT and control. The participants in the G-IPT group received 16 sessions of treatment, whereas the participants in the control group did not receive any intervention. All participants completed the assessment three times: before, after, and tracking. Results: The results showed that the total score and the scores of all subscales of aggression dropped significantly $(\mathrm{P}<0.01)$. Also, the total score and the scores of objective support and utilization degree of social support increased significantly $(\mathrm{P}<0.05)$. Conclusion: $\mathrm{G}-$ IPT was effective in treating aggression and improving social support level, and the effect was stable.
\end{abstract}

Keyword: Aggression; Chinese university students; Group interpersonal psychotherapy; Social support 
